\title{
Law and Psychology, Complementary Sciences in the Latin American Oral System
}

\author{
José Paulino Dzib Aguilar, Carlos Alberto Macedonio Hernández \\ Autonomous University of Yucatan (UADY), Yucatán, Mexico \\ Karime Esther Medina Farah \\ Clinic in Therapeutic Justice of Yucatan (CIIJUTEY), Yucatán, Mexico \\ Lucely Martina Carballo Solís \\ Autonomous University of Yucatan (UADY), Yucatán, Mexico
}

\begin{abstract}
Psychology and the law share important similarities in dealing with antisocial behavior, allowing the interdisciplinary work in the new justice system that is used in Mexico, which is very important to measure the impact of the crime on the victim and establish with this analysis the amount of the damage repair as well as the degree of punishability, there is also many disciplines as victimology, which necessarily require the intervention of psychology and law to study the victim of a crime. Psychology is a social science that seeks to explain and to modify the human behavior; on the other hand, law deals with social coexistence, regulating some of those human behaviors through laws and codes which are essential to achieve that peaceful coexistence between people; both disciplines are social sciences that study the human behavior each in their field of knowledge
\end{abstract}

Keywords: psychology, law, administration of justice, principles

\section{Introduction}

We cannot forget that psychology and law belong to a same branch of knowledge, human, and social sciences, and that both have a common objective: the human behavior; these similarities lead to rethinking the ethical exercise in forensic psychology, which is a branch of psychology which works as auxiliary to the process of administration of justice in the courtrooms.

According to the approach of the American Psychological Association (2013), psychologists are at the mercy of the laws of the place where they practice professionally, so the professionals involved in this area need to be constantly updated on laws, codes, and standards, both local and national, that rule in the States.

There are different resources to link both sciences in the professional practice. This article aims to explain

José Paulino Dzib Aguilar, Doctor in Social Problems, professor, College of Psychology, Autonomous University of Yucatan (UADY), Yucatán, Mexico.

Carlos Alberto Macedonio Hernández, Juris Doctor, professor, College of Psychology, Autonomous University of Yucatan (UADY), Yucatán, Mexico.

Karime Esther Medina Farah, Bachelor of Psychology, psychologist, Clinic in Therapeutic Justice of Yucatan (ClIJUTEY), Yucatán, Mexico.

Lucely Martina Carballo Solís, Master in Law, professor, College of Psychology, Autonomous University of Yucatan (UADY), Yucatán, Mexico. 
the complementarity of psychology and the law, as well as propose a methodological instrument to organize the information obtained during the expert evidence process.

\section{Contrasts and Complementarity Between Both Sciences}

As we can contrast the practices of psychology and law in its search for regulating human behavior, both designate procedures that share similar in their objectives. Table 1 describes the procedures and the similarity of their objectives:

Table 1

Procedural Similarities Between Psychology and Law

\begin{tabular}{lll}
\hline Psychology & Law & Objective \\
\hline Diagnosis & Sentences & Expert report \\
Classification & Typification & Categorization \\
Therapy & Mediation/detention & Intervention \\
Diagnose manuals & Codes & Normativity \\
Principles & Laws & Guidelines \\
Methodology & Due process & Systematization \\
\hline
\end{tabular}

In psychology, as in the law, expert reports are produced. Psychologists prepare a diagnosis after having measured, evaluated, and assessed the behavior. On the other hand, in the legal field, sentences are pronounced, as judgments following the evaluation of the evidence presented during all the process. As it can be seen, both fields talk about a result at the conclusion of a certain stage of the process, and they use their own terminology according to the profession.

Both sciences organize, or divide a set of elements in categories or groups by making classifications. Regarding the people and type of disorders, this is an exercise that belongs to psychology; whereas the professionals of the law typify the behavior according to a pattern, a model, or a norm. In the intervention to modify behaviors, psychology uses therapy as the strategy; lawyers make these interventions through mediation and/or imprisonment.

To achieve these rankings and interventions appropriately, the practice of both disciplines are based on laws and codes, in the case of lawyers, and protocols and manuals in the case of psychologists; the later ones use different tools, such as Diagnostic manuals, which serve as documentary instruments where the classifications of mental disorders are registered, such as the DSM-5 and ICD-10. Lawyers, for example, use codes such as the criminal code, the civil code, the family code, and among others.

There are principles governing the explanations of behavior while laws rule social behavior. Finally, both sciences are systematized methodologically; in psychology, the principles for scientific praxis is called methodology, whereas in law is called due process, that is the way in which justice is administered.

As it can be seen, both sciences share similar strategies, procedures, and objectives.

\section{Considerations of the Professional Practice of Psychology the Field of Law}

The practice of psychology must redirect its interventions in the professional praxis, so that they do not put the rights of the people, nor the professional practice at risk. Some of these risks are: the duplicity of roles, the use of the informed consent, erroneous psychopathological and forensic diagnostics process, using inadequate instruments and therapeutic interventions, as well as the lack of knowledge of the new adversarial system 
established in Mexico, in the Constitutional Reformation of the year 2008, which has been gradually implemented in the country.

There is a paradigmatic change in the principles for the evaluation of evidence, such as healthy criticism, the maximum experience, and the logical principles. These are the basis to ensure a more accurate reasoning in the administration of justice, when deciding on the penal responsibility of a person who committed a crime and who is under a trial. On the other hand, the legal and forensic psychologists must understand and apply these principles in their interventions because they provide support to the scientific paradigm in the administration of justice (Gómez González, 2016).

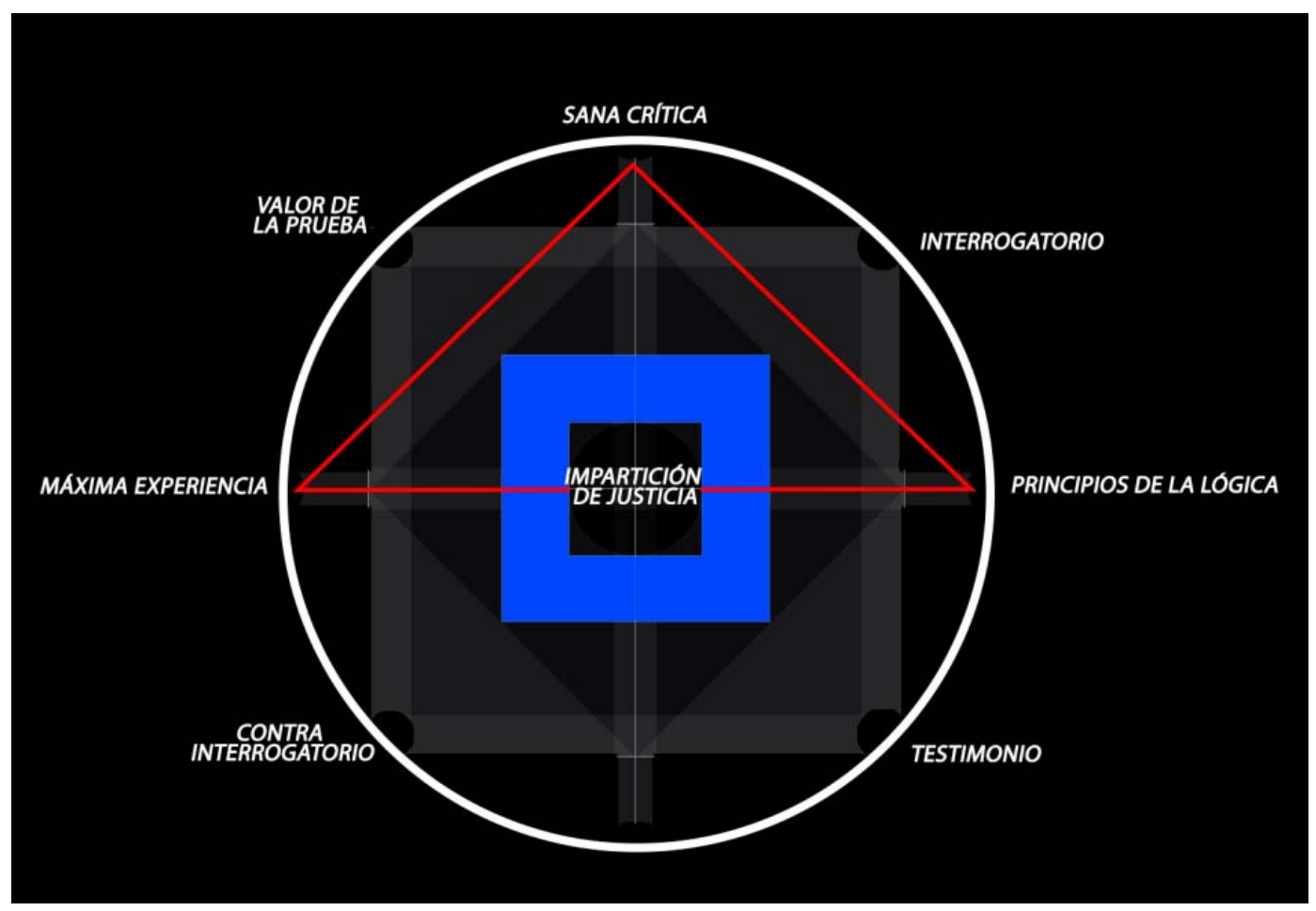

Figure 1. The framework of administration of justice.

The healthy criticism, mentioned previously, is the intellectual exercise made by the judge seeking for a suitable appreciation of results of the evidence. It has been defined as the interpretive logic and the common feelings of the people (Aroca, 2002).

In other words, healthy criticism is the method of appreciation of the evidence, where the judge will value it according to the logic, the principle of the experience and the scientifically strengthened knowledge (Fuchslocher, 2010). It is a tool that the judges must use, at the level of logic to evaluate the evidence, in order to motivate and support their decisions, while the experts in legal and forensic psychology must also support the decisions expressed in their reports. It includes the null hypothesis and the alternative hypothesis. The aim is to achieve certainty between the possibilities of the behavior because in exercising criticism a conclusion is reached, and the expert chooses the possibility that is more suitable to the given situation (Fuchslocher, 2010; Plascencia Villanueva, 1995).

These arguments must be coherent and in agreement with the interrogation, that is why the principle of the logic is applied, that is the formal logic, which is applied through its own principles and serve as the rational 
part in the judicial decision, these are the principle of identity, contradiction, excluded middle and sufficient reason (Barrios González, s.f.). Another consideration in the practice of psychology and law nowadays is the need for systematization as to decide who accompanies an alleged victim in his or her statement, or in a judicial process, because such support is often requested for legal proceedings. The purpose of this systematization is to ensure that the person who accompanies the alleged victim is suitable, complying with certain standards, and with the necessary knowledge for adequate performance.

To systematize a procedure means to have a methodology that ensures the validity and reliability of the process. Validity is met when the right companion for the alleged victim is chosen. The companion must be an expert or a person who reduces the possibility of repeat victimization. Reliability means that every time that the systematization is applied, it would be confirming that the person who was chosen was adequate for the accompaniment (Dzib-Aguilar \& Medina-Farah, 2018).

The professionals working in this field must deal with the victims in a gentle, kind, and calmed manner, in order to develop confidence; such factors will affect the predisposition of the the children and adolescents to render a statement. To decide what professional or family member would be accompanying the victim, requires to take into consideration several aspects of their environment. These are the memory, evolutionary development, suggestibility and dynamics in which their family environment functions; the labor environment or even the judicial system and the administration of justice (Dzib-Aguilar \& Medina-Farah, 2018).

It is necessary to evaluate if the memory of the person is intact regarding the presence or absence of neurological damage, mental disorder, or if the person is in an exasperated state of mind that could contaminate the memory at the time of rendering a statement, as well as the type of memory that the victim uses. As far as the evolutionary development aforementioned, it is required to know, according to their age, the cognitive, social, and interpersonal development expected in the subject, in order to evaluate if he/she is apt or not to render his/her statement. Similarly, to know about the emotional state and the mourning state of the NNA at the time of rendering his/her statement, is in order to perform an efficient intervention and reduce the emotional effects in the alleged victim. Regarding the suggestibility, it is necessary to assess whether the alleged victim has some link that is influencing objectivity in order to obtain the facts in the most faithful manner in which they occurred (Dzib-Aguilar \& Medina-Farah, 2018).

This proposal of systematization is an aspect that has not been defined nowadays, and from the psychological standpoint, it influences, to a great extent, the rendition of a statement.

\section{Methodological Consistency}

Do legal and forensic psychologists use a reference framework that is consistent with the instrument and the form in which they interpret the results? The principle of logics seeks to answer this question. Logics imply the methodological process to be followed as the theoretical frame aimed at the consistency between instruments and the way to interpret them. Since the logic in the methodology reduces the subjectivity of the interpretation, there is greater control in the practice and it is based on a systematic procedure.

As it was mentioned earlier, healthy criticism also involves psychology, which is understood as the inner element that rules the life of the people and which is evident through facts of knowledge, feelings, and will. The lawyers and judges must include this aspect in the evaluation of the evidence without forgetting the principle the experience, also known as common experience, which include the lessons acquired with the use and the practice, integrating common sense, and which are found in each person. It comprises the qualifications by 
which a professional grounds his or her knowledge including certificates, research, scientific data, as well as the bibliography that has been used, considering that the professional acts as an agent.

The professional intervention, derived from the previous elements, must be present in all the stages of the Accusatory Penal Process accordingly, the preliminary stage or investigation, the intermediate stage, and finally the stage of oral judgment or debate (Procuraduría General de la República, 2017).

In the preliminary stage or investigation of the facts, any evidence must be successfully obtained, including the statements, Therefore, it is necessary to follow a protocol to obtain the first statement, which should be carried out by an interdisciplinary team that aimed at having the most appropriate procedure and with the least contamination possible, and taking into account, during the interrogation , certain aspects, such as the type of memory used, personality of the subject, culture, etc., in order to determine the most adequate interrogation method and to validate the information obtained that way.

After the investigation stage, the intermediate stage will follow, what includes the discovery, proof, and validation of evidences before the judge in the same hearing. During the stage of oral judgment, the prosecution and the defense present their evidence before a tribunal. Both defense and prosecutor can debate over the evidence submitted, which can be documents or statements. Such debate will serve as the basis for the solid argumentation in order to support the final statement at the end of the hearing. In this stage, as well as in the previous one, the professionals must consider the importance of memory. This is an extremely important aspect, and it is normally ignored by the professionals involved in the process.

As the judicial process progresses, the memory could be modified due to the contamination generated within it. Hence, it is necessary to know what type of memory is used, according to the age and characteristics of the parties and thus to be able to evaluate the statements obtained and make appropriate comments. This is especially relevant when minors are involved due to the conditions in which they are in, according to their evolutionary development (Gómez González, 2016).

\section{Proposal of a Methodological Instrument}

When making expert psychological forensic reports, the following format is proposed, which we have called "forensic evidence".

Forensic evidence, in the psychological evaluation, is an organizer to demonstrate the relation, that skillful psychologists make, between their findings obtained by their techniques and instruments, and the evidence recorded in the investigation folder, which helps the judge to see the relation between the psychological signs of social or antisocial behavior, and the different evidence presented.

When making expert psychological test, the following format is proposed, which we have called "forensic evidence".

Table 2

Forensic Evidence

\begin{tabular}{lll}
\hline Relevant information & Source of information & Relation psychology-evidence \\
\hline & e.g., Interview of the mother by the & \\
e.g., The girl reports fear, tension, & psychologist & Statement where the girl describes the \\
nervousness and weeping due to... & Date: ... & Place: ... \\
\end{tabular}


The format will give greater clarity to the evidence, by presenting the most important extract of the evidence regarding the objectives. By presenting the information more accurately the evidence will be easier to understand.

Continuing with the stages of the accusatory penal process, the intermediate stage, or preparation of the oral judgment, ends after the judge of control has declared the legality or constitutional guarantees and the ruling or resolution to open the oral trial, and then, the last phase of the ordinary process, commonly, known as “stage of oral trial or debate”, begins (Gómez González, 2016).

There are international, national, and local laws and norms that rule the judicial process for all the participating professionals. The forensic legal psychologist will have to abide by and to fulfill the legal responsibilities appertaining to it according to the penal, civil and familiar codes, the protocols of action of the Supreme Court of Justice of the Nation, as well as the guidelines proposed by the American Psychological Association (APA) regarding the professional practice and the codes and protocols thereto appertaining to the specifications of the case.

\section{Conclusions}

The psychological praxis in a judicial process is governed by laws, codes, and protocols of action which guide the participation of the professional, and lay down the penalties for their lack of compliance. There is certain knowledge, in both disciplines, which psychologists and lawyers practicing in these areas should have. An adequate interdisciplinary work is very important in order to provide their services in the most competent way and of the best quality possible.

As it can be seen, both sciences share similar strategies and procedures with the specificity of each profession. Any process, psychological as well as legal, must have a methodology that complies with the validity and reliability of the process. To know the laws, codes, and protocols appertaining to an adequate professional exercise of the forensic psychology is obligatory. Likewise, the lawyer must understand the methodology used by the forensic psychologist in order to prepare a solid and valid case for his/her closing arguments and to obtain a favorable sentence.

The fundamental aspect of both disciplines is the use of the scientific paradigm which guides the procedures or the administration of justice in the penal oral systems, which draw us to a more objective reality and thus reducing the probability of error.

\section{References}

American Psychological Association. (2013). Specialty for guidelines forensic psychologists. Washington, DC: Author. Aroca, J. M. (2002). La prueba en el proceso civil. Civitas.

Barrios González, B. (s.f.). Teoría de la Sana Crítica. Retrieved from http://www.academiadederecho.org/upload/biblio/contenidos/Teoria_de_la_sana_critica_Boris_Barrios.pdf

Dzib Aguilar, J. P. (2017). Cuadro Impartición de Justicia. Work presented in the X International Conference of Legal and Forensic Psychology, Seville, Spain.

Dzib Aguilar, J. P., \& Medina Farah, K. E. (2017). Forensic evidence work presented in conference "Professional Secrecy Versus Legal Responsibilities”. Yucatan, Mexico: University Anáhuac Mayab.

Dzib-Aguilar, J. P., \& Medina-Farah, K. E. (2018). Systematization to decide who accompanies an alleged victim during his or her statement or a judicial process (Unpublished theies, University Anáhuac Mayab, Yucatan, Mexico).

Fuchslocher, A. V. (2010). La sana crítica. Santiago, Chile: Facultad de Derecho, Universidad de los Andes.

Gómez González, A. (2016). Reforma Penal 2008-2016. El Sistema Penal Acusatorio en México. Retrieved from http://www.inacipe.gob.mx/stories/publicaciones/novedades/ReformaPenal2008-2016.pdf 
Plascencia Villanueva, R. (1995). Los medios de prueba en materia penal. Boletín Mexicano de Derecho Comparado Nueva Serie Año XXVIII.

Procuraduría General de la República. (2017). Cuáles son las etapas de un proceso en el Sistema de Justicia Penal? Retrieved from https://www.gob.mx/pgr/articulos/cuales-son-las-etapas-del-proceso-en-el-sistema-de-justicia-penal

Rodriguez Ortega, G. (s.f.). Introducción a la psicología y el derecho. Retrieved from http://www.juridicas.una 